\title{
PIXEL LEVEL DATA FUSION OF MARS EXPRESS HRSC LINEAR PUSHBROOM IMAGERY BASED ON THE RIGOROUS GEOMETRIC MODEL
}

\author{
Xun Geng a,b,c,*, Qing Xu ${ }^{\mathrm{a}}$, Yuange Lan ${ }^{\mathrm{b}}$, Yifan $\mathrm{Hou}^{\mathrm{a}}$, Shuai Xing ${ }^{\mathrm{a}}$, Chaozhen Lan $^{\mathrm{a}}$ \\ ${ }^{a}$ Institute of Geographic Space Information, Information Engineering University, Zhengzhou 450052, China - \\ gengxun.rs@gmail.com \\ ${ }^{\mathrm{b}} \mathrm{Xi}$ 'an Institute of Surveying and Mapping Information Technology, Xi'an 710054, China- \\ ${ }^{c}$ State Key Laboratory of Geo-information Engineering, Xi'an 710054, China
}

\section{Commission VII WGVII/6}

KEY WORDS: Mars Express; HRSC; Image Fusion; Linear pushbroom imagery; back projection; best scan line searching

\begin{abstract}
:
The High Resolution Stereo Camera (HRSC) on European spacecraft Mars Express is especially designed for topographic mapping of Mars. HRSC can acquire both panchromatic and multi-spectral images simultaneously. The resolution of panchromatic image is higher than that of multi-spectral image. Through image fusion, the high resolution multi-spectral image of Mars can be generated. In this paper, the HRSC raw image is geometrically rectified firstly based on rigorous geometric model. Then, the panchromatic image and multi-spectral image are fused based on IHS transformation. In order to improve the processing efficiency, a fast algorithm for back projection of ground points based on the geometric constraints of the central perspective planar of scan line is proposed. Experiments tested on HRSC images demonstrate our algorithm's feasibility and efficiency.
\end{abstract}

\section{INTRODUCTION}

Deep space exploration has great value for civilization and scientific research. Mars Express is European's first Mars exploration mission. The High Resolution Stereo Camera (HRSC) mounted on Mars Express is especially designed for topographic mapping of Mars. HRSC has the merits of both high resolution and global coverage(Kirk, 2000; Albertz, 2004; Albertz, 2005). The HRSC camera is designed as a multi-line pushbroom scanner with nine CCD line detectors mounted parallel in the focal plane. Five panchromatic images and four multi-spectral images can be acquired simultaneously. A high GSD of up to $10 \mathrm{~m} /$ pixel can be acquired when the orbit height is $250 \mathrm{Km}$. Currently, images acquired by HRSC has almost covered the whole Mars surface. The focal length of HRSC is $175 \mathrm{~mm}$ and every scan line has 5184 pixels. Table 1 shows the technical parameters of HRSC camera.

\begin{tabular}{|c|c|}
\hline Camera Parameter & Parameter \\
\hline Channels & 9 \\
\hline Pan Image angles & $0^{\circ} \pm 18.9^{\circ} 、 \pm 12.8^{\circ}$ \\
\hline Multi-spectral channel & near-infrared, red, green, blue \\
\hline Focal length/mm & 175 \\
\hline Pixel size/um & 7 \\
\hline IFOV/arcsec & 8.2 \\
\hline Image size & 5184 pixels/line \\
\hline Radiometric resolution/bit & 12 \\
\hline Orbit height $/ \mathrm{km}$ & 250 \\
\hline Image resolution/m & 10 \\
\hline $\mathrm{B} / \mathrm{H}$ & $0.68 / 0.45$ \\
\hline
\end{tabular}

Table 1. Geometric parameters of HRSC camera
The coordinates of HRSC is defined as follows. The $Y$ axis is parallel to flight direction and the $X$ axis is along the CCD line array which is perpendicular to the $Y$ axis, while the $Z$ axis is perpendicular to focal plane and points to image line direction. The $X Y Z$ constitute right hand system.

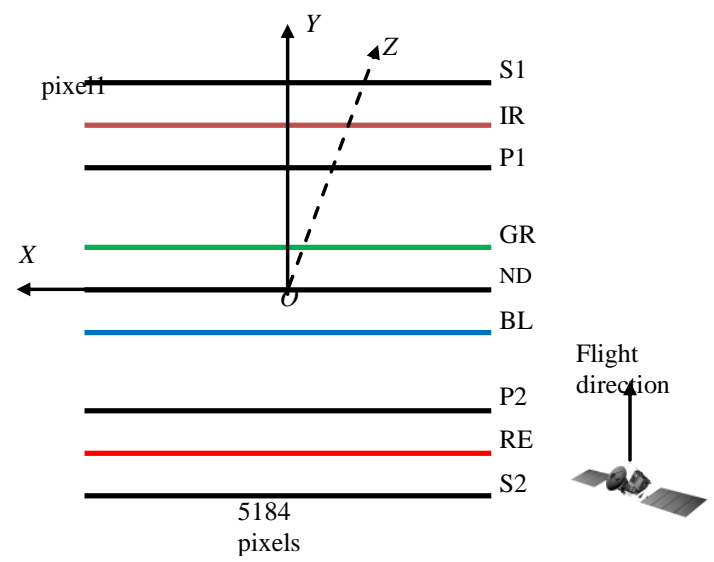

Figure 1. Line array arrangement of HRSC camera

The panchromatic image of HRSC has the merit of high spatial resolution but it is lack of spectral information. While the multispectral image of HRSC has the merit of abundant spectral information, whereas it has a low spatial resolution. Therefore, a high spatial resolution multi-spectral image can be fused. Before the image can be fused, panchromatic and multi-spectral image of HRSC need to be geometrically rectified firstly. In order to get high accuracy and proper registered images, the rigorous geometric model is adopted in image rectification. The Level 2 data of HRSC which pertain the original geometric

\footnotetext{
* Corresponding author.
} 
information is used to generate digital orthography. After geometrically rectified, the panchromatic and multi-spectral image should be registered properly which is essential for image fusion. The camera interior and exterior orientation parameters of HRSC can be acquired from SPICE kernels. The time and datum conversion is illustrated in figure 2 .

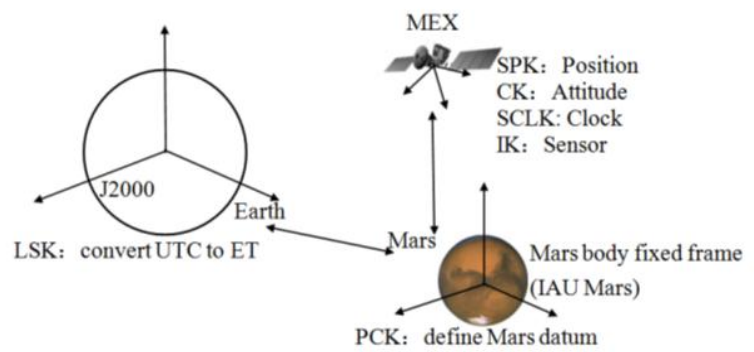

Figure 2. Transformation of time and coordinates using SPICE kernels

\section{IMAGE FUSION OF HRSC IMAGERY}

Remote sensing image fusion can be classified into three classes. They are pixel level, feature level and decision level. In this paper, the pixel level image fusion of Mars Express HRSC image is implemented through IHS transformation based on the rigorous geometric model of line array scanners. IHS color system which used intensity, hue and saturation to represent color is different from the RGB color system. The image fusion procedure of HRSC image is given in figure 3 .

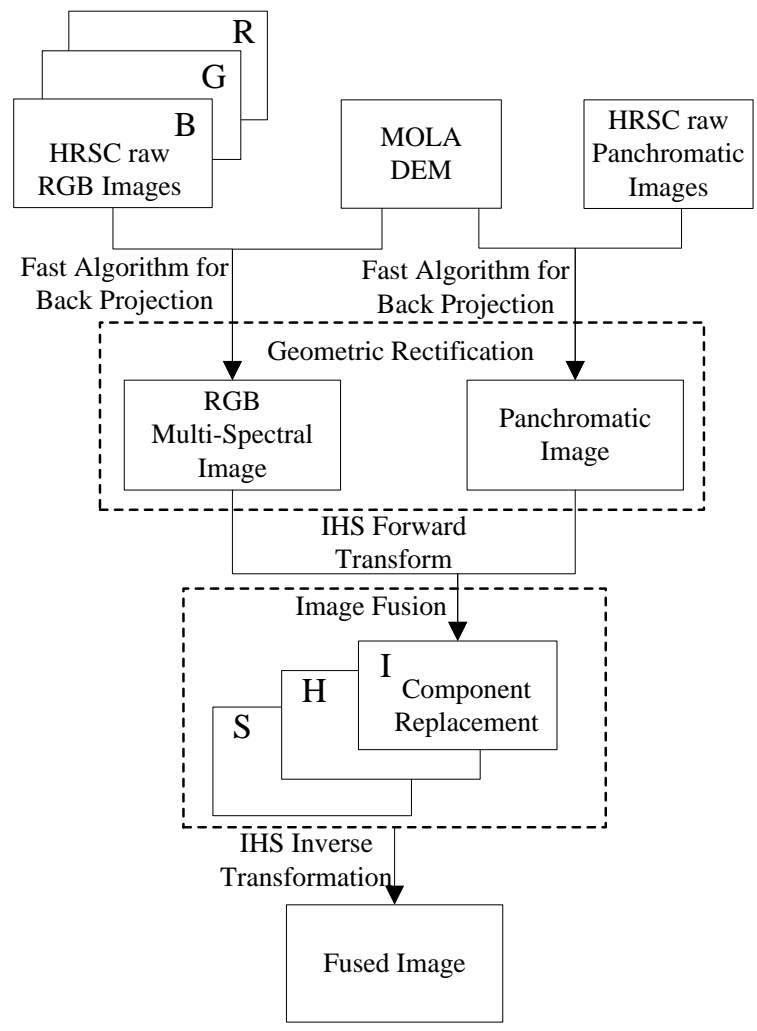

Figure 3. Image fusion procedure of HRSC imagery

The procedure of image fusion of Mars Express HRSC image through IHS transformation is as follows.
(1) Firstly, the original HRSC linear array pushbroom image is rectified based on rigorous geometric model and the high resolution panchromatic image and true color image is generated. MOLA DEM whose grid space is about 500 meters can be used to assist rectification when there is no high precision DEM.

(2) Then the true color RGB image is resampled and a new image with the same image size and spatial resolution as the panchromatic image is generated.

(3) Thirdly, the true color RGB image is converted into IHS color space through IHS forward transformation.

(4) The intensity component of the true color image in IHS color space is replaced by the pixel value of the panchromatic image.

(5) The high resolution true color image is derived through IHS inverse transformation.

\section{FAST ALGORITHM FOR BACK PROJECTION}

Currently, photogrammetric processing of linear pushbroom imagery is a hot spot(Geng, 2011).The basis of image fusion of HRSC linear pushbroom image is geometric rectification. Because of the special imaging principle of line array scanners, the back projection of ground points needs multiple iterations to find the best scan line(Zhao, 2011). The traditional method for back projection is performed through collinearity equation computation with dozens of iterations which is not feasible for practical use. Because of the huge amount data of HRSC imagery and the complicated computation of back projection, if the traditional back projection method is used to perform image rectification, the image fusion processing efficiency will be very slow. In this paper, a fast algorithm for back projection of ground points is proposed based on central perspective plane of scan line. As experiment results demonstrate, based on our fast back projection algorithm, the image fusion processing efficiency will be improved greatly.

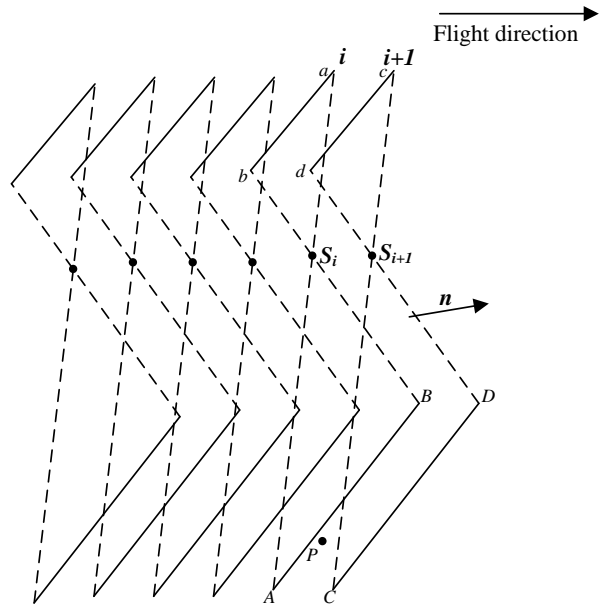

Figure 4. Fast algorithm for back projection

The basic principle of our fast algorithm for back projection is using the geometric constraints of central perspective plane of scan line in ground space (see figure 4), a central perspective plane of scan line can be formed by the line CCD array and the corresponding perspective center, a ground point $P$ lies between the $i$ th central perspective plane and the $i+1$ central perspective plane. The scan line which is nearest to the ground point $P$ can be quickly determined through the computation of the distance between the ground point $P$ and each central perspective plane 
of scan line. It is easily seen that our algorithm which is based on the geometric constraints in ground space can determine the best scan line with less iterations and the computation is just simple analytical geometry operations. Consequently, our algorithm performs better than the traditional method.

The procedure of our fast algorithm for back projection is as follows.

(1) Acquire the geometric parameters of line CCD array, exterior orientation parameters to construct the rigorous geometric model for HRSC.

(2) Compute the plane equation of central perspective plane of each scan line.

$$
A x+B y+C z+D=0
$$

Where $A, B, C$ is the direction number of normal line of the central perspective plane. The distance between the ground point $M\left(x_{0}, y_{0}, z_{0}\right)$ and the central perspective plane is computed.

$$
d=\frac{\left|A x_{0}+B y_{0}+C z_{0}+D\right|}{\sqrt{A^{2}+B^{2}+C^{2}}}
$$

(3) The scan line nearest to Ground $P$ can be estimated through the distance between each central perspective plane.

(4) The distance of ground point $\mathrm{P}$ to the $i$ th central perspective plane is computed iterately until the distance is shorter than the distance between two neighbouring central perspective plane.

(5) Finally, the best can line can be accurately determined

$$
L=i+\frac{D_{i}}{D_{i}+D_{i+1}}
$$

\section{EXPERIMENT AND ANALYSIS}

Creater and valley area of Mars is selected to perform our experiment and the basic information about our test data is illustrated in Table 2. The experiment environment is based on Windows XP operating system and the source code is

\begin{tabular}{|c|c|c|c|c|c|}
\hline \multirow{2}{*}{$\begin{array}{l}\text { Terrain } \\
\text { type }\end{array}$} & \multicolumn{2}{|c|}{$\mathrm{GSD}(\mathrm{m})$} & \multicolumn{2}{|c|}{ image size } & \multirow{2}{*}{$\begin{array}{l}\text { Time } \\
\text { (s) }\end{array}$} \\
\hline & Pan & RGB & Pan & RGB & \\
\hline Valley & 20 & 80 & $\begin{array}{l}9664 \times \\
62304 \\
\end{array}$ & $\begin{array}{l}2416 \times \\
15576\end{array}$ & 1769 \\
\hline Creater & 15 & 30 & $\begin{array}{l}4640 \times \\
20232\end{array}$ & $\begin{array}{l}2320 \times \\
10116\end{array}$ & 281 \\
\hline
\end{tabular}
developed with Visual Studio 2008. The CPU is Intel Core i7 $(3.4 \mathrm{GHz})$ and the memory size is $3.24 \mathrm{~GB}$.

Table 2. The basic formation of test data

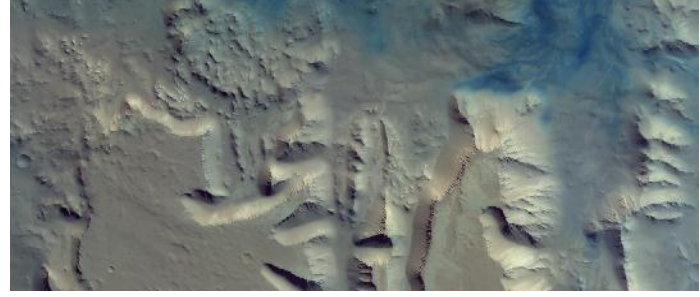

(a)Fused RGB Image (full image)

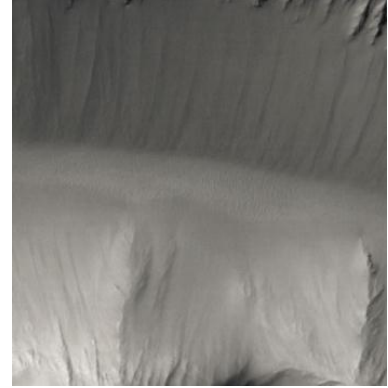

(b)Pan

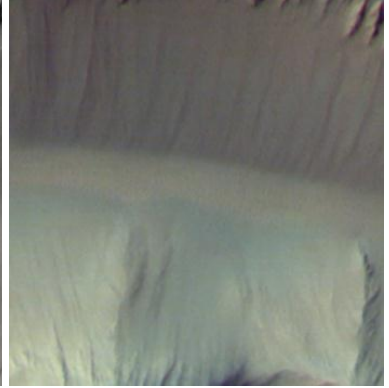

(c) RGB

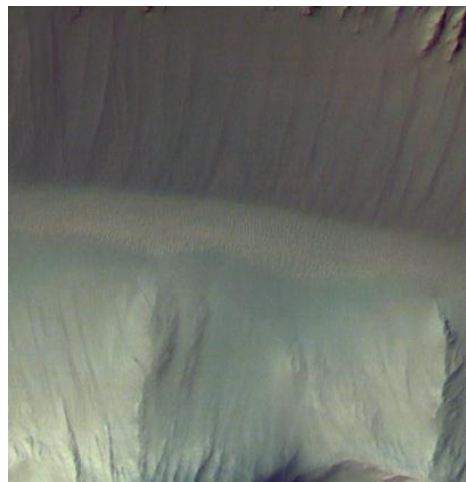

(d) Fusion results (local image)

Figure 5. Image fusion results of test 1

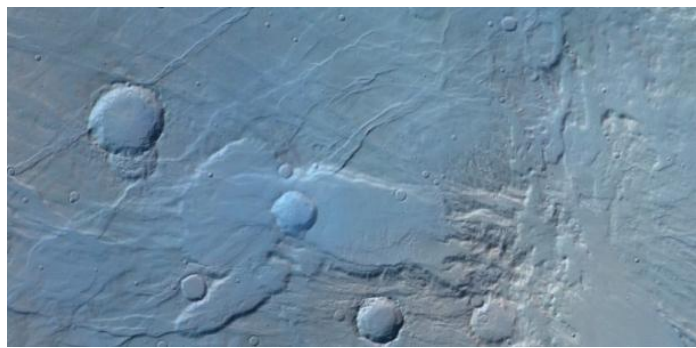

(a)Fused RGB Image (full image)

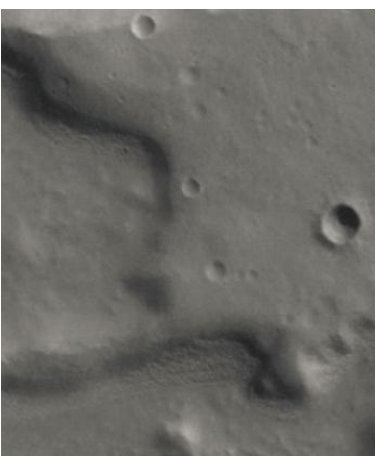

(b)Pan

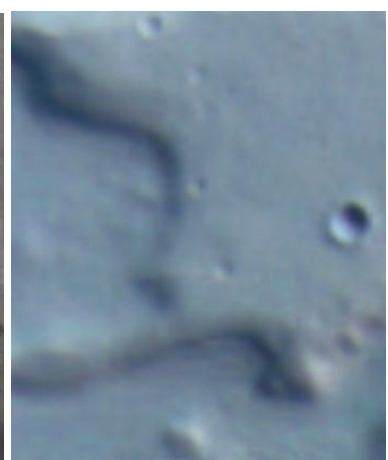

(c) RGB 
International Archives of the Photogrammetry, Remote Sensing and Spatial Information Sciences, Volume XL-7/W1, 3rd ISPRS IWIDF 2013, 20 - 22 August 2013, Antu, Jilin Province, PR China

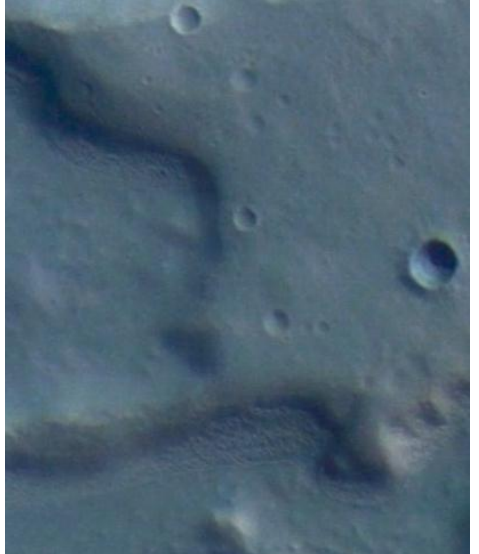

(d) Fusion results (local image)

Figure 6. Image fusion results of test 2

From the experiment results we can draw conclusion as follows. (1)A new high spatial resolution RGB image can be generated through image fusion of panchromatic image and true color image of HRSC. Therefore, with HRSC's global coverage ability, a beautiful and colorful Mars digital orthography which covers the whole Mars surface can be derived.

(2)When the mage size is greater than several hundred MB, the image fusion can be performed within several minutes. Experiment results demonstrate that our algorithm show better feasibility.

\section{CONCLUSION}

The high spatial resolution topographic data and multi-spectral image data is the basis for Mars research. In this paper, the image fusion of Mars Express HRSC linear array pushbroom imagery based on the rigorous geometric model is researched. With our fast back projection algorithm the image fusion method of HRSC show great feasibility.

\section{ACKNOWLEDGEMENTS}

This paper is funded by the National Basic Research Program of China (973 Program) (2012CB720000) and State Key Laboratory of Geo-information Engineering of China.

\section{REFERENCE}

Kirk R.L., Kraus E.H., Rosiek M., 2000. Recent Planetary Topographic Mapping at the USGS, Flagstaff: Moon, Mars, Venus, and Beyond. In: The International Archives of the Photogrammetry, Remote Sensing and Spatial Information Sciences, Amsterdam, pp. 476-490.

Albertz J., Gehrke S., Wahlisch M., 2004. Digital Cartography with HRSC on Mars Express. In: The International Archives of the Photogrammetry, Remote Sensing and Spatial Information Sciences, Istanbul, pp. 869-874.

Albertz J., Attwenger M., Barrett J., 2005. HRSC on Mars Express-Photogrammetric and Cartographic Research. Photogrammetric Engineering \& Remote Sensing, 71(10), pp. 1153-1166.

Geng Xun, Xu Shuiping, Gong Zhihui, Yuan Jun, 2011.The Data Processing and Accuracy Analysis Method of Aerial
Triangulation for ADS40 Linear Array Images. Geomatics and Information Science of Wuhan University, 36(7), pp. 776-779.

Zhao Shuangming, Li Deren, Mou Lingli, 2011. Inconsistency Analysis of CE-1 Stereo Camera Images and Laser Altimeter Data. Acta Geodaetica et Cartographica Sinica, 2011, 40(6), pp.751-755. 